\title{
Effect of ultrasound-guided percutaneous neuromodulation applied to the femoral nerve on pain and range of motion in patients with anterior knee pain: A case study
}

\author{
Paula García-Bermejo ${ }^{1}$ Carlos Romero-Morales ${ }^{2}$ (i) \\ ${ }^{1}$ DINAMIA Physiotherapy Clinic, Madrid, Spain \\ 2 Facultad de Ciencias del Deporte, Universidad Europea de Madrid, \\ Villaviciosa de Odón, Madrid, Spain \\ ${ }^{3}$ Departamento de Fisioterapia, Universidad de Sevilla, Sevilla, Spain
}

Blanca de-la-Cruz-Torres 3 (i)

\begin{abstract}
Address for correspondence Blanca de-la-Cruz-Torres, Department of Physiotherapy, University of Seville, Avicena street, s/n, 41009, Seville, Spain (e-mail: bcruz@us.es).
\end{abstract}

\begin{abstract}
Keywords

- anterior knee pain

- femoral nerve

- percutaneous neuromodulation

- ultrasound

Introduction Anterior knee pain (AKP) is one of the most frequent reasons for physical therapy consultations, remaining a difficult treatment challenge for professionals. The aim of this study was to evaluate the effects of an intervention using ultrasound-guided percutaneous neuromodulation (US-guided PNM) applied to the femoral nerve on pain and knee flexion range of motion (ROM), in patients with unilateral chronic anterior knee pain.

Methods Eight patients received a single intervention of NMP-e on the femoral nerve of the symptomatic knee. The level of pain and ROM were measured before, immediately after and 24 hours after the intervention.

Results A decrease in pain and an increase of ROM were observed after the intervention and at 24 hours. For pain values, a medium effect size was observed ( $E S=0.63 \pm 0.43)$ after the intervention, and a large effect size $(E S=1.73 \pm 0.44)$ was found at $24 \mathrm{~h}$. Concerning ROM, the effect size was large at both moments (ES= $-1.30 \pm 1.14 ;-1.76 \pm 0.80$, respectively).

Conclusion A single intervention of US-guided PNM on the femoral nerve produces a decrease of pain and increases the ROM, which is greater $24 \mathrm{~h}$ after the stimulation. Level of Evidence Level II-3.
\end{abstract}

\section{Introduction}

Anterior knee pain (AKP) is one of the most frequent reasons for physical therapy consultations, remaining a difficult treatment challenge for professionals. It is characterized by a non-specific knee pain located around the patella, mainly anteromedial and/or retropatellar. ${ }^{1}$

The etiology of AKP is unclear, although it is suggested to be multifactorial, ${ }^{2,3}$ including patellar anomalies, ${ }^{4}$ biomechanical disorders ${ }^{5}$ or overuse, especially among teenagers. ${ }^{6}$ However, different authors ${ }^{7}$ have suggested that AKP is not only a local

received

November 2, 2019

accepted

December 5, 2019
DOI https://doi.org/

10.1055/s-0040-1701431. ISSN 2386-4591. mechanical pain, but rather the result of sensitization of the neural system, likewise, this occurs in other musculoskeletal pathologies. De-la-Cruz-Torres ${ }^{8}$ demonstrated that patients with unilateral chronic epicondylalgia had a decreased excitability of the radial nerve, independent of the symptomatic elbow. Rodríguez-Fernández et $\mathrm{al}^{9}$ reported that patients with functional ankle instability displayed a decreased excitability of the common peroneal nerve, compared with subjects without ankle lesions. Regarding the knee, Lin et $\mathrm{al}^{7}$ found that patients with AKP presented an alteration in the mechanosensitivity of the femoral nerve. As a result, many therapists
Copyright (e) 2020 by Thieme Revinter

Publicações Ltda, Rio de Janeiro, Brazil
License terms

$\Theta(1) \Theta \circledast$ 
include clinical tests of peripheral nerves within their assessment protocols of AKP.

The electric stimulation of the peripheral nerve is a therapeutic procedure which applies an electrical current on the peripheral nerve in order to treat chronic pain. ${ }^{10,11}$ The electric impulses interfere with the action of the impulses transmitted by the central nervous system, which make the person feel pain. At present, the use of ultrasoundguided percutaneous neuromodulation (US-guided PNM) on the peripheral nervous system is on the rise. This minimallyinvasive intervention consists of the application of a percutaneous electric stimulation via an acupuncture needle which is placed close to the nerve (perineurium) or the motor point of the muscle, under ultrasound guidance. ${ }^{12}$ Mainly, US-guided PNM is used for therapeutic purposes, such as the relief of chronic pain ${ }^{13}$ and neuropathic pain ${ }^{14}$ or with the aim of improving muscle activity. ${ }^{15}$ Regarding chronic pain, different authors ${ }^{7}$ justify the application of neuromodulation due to mechanisms of central sensitization. However, there is still limited evidence regarding the effectiveness of physical therapy techniques directed at chronic pain affecting the peripheral nerves for the treatment of different musculoskeletal pathologies, especially in more chronic cases.

Therefore, the aim of this study was to evaluate the effects of an intervention of US-guided PNM on the femoral nerve on variables of pain and knee flexion range of motion (ROM) in patients with unilateral AKP.

\section{Methods}

In total, eight patients (six women, two men) with unilateral AKP were recruited from a private clinic. The subjects received an intervention of US-guided PNM on the femoral nerve of the affected side. The inclusion criteria were as follows: presenting unilateral knee pain, duration of pain greater than three months and aged between 25-55 years old. The exclusion criteria were: presenting a structural lower limb length discrepancy, surgery on any of the knees, lumber spine pathology (protrusion, herniated disk,...) due to the possible involvement of the lumbar plexus, ${ }^{16}$ being under medication and the specific contraindications for US-guided PNM (needle phobia, pregnancy, pacemaker, epileptic seizures). ${ }^{12}$

\section{Ethical Considerations}

This study was approved by the local ethics committee (08/ 2008) and fulfilled all the principles established in the Helsinki Declaration. All the subjects signed the informed consent in writing to participate in this study.

\section{Variables}

The demographic data were obtained, including age, weight, height, body mass index (BMI), sex, dominant side, symptomatic side, and duration of symptoms. The clinical variables analyzed were:

- The level of pain reported by the patient was evaluated using a visual analog scale (VAS). ${ }^{17}$ To do so, a ruler measuring $100 \mathrm{~mm}$ was used with two labels, "no pain" and "maximum pain", located on either side of a line, and the patient had to indicate their level of pain with a vertical line ( 0 , no pain; $100 \mathrm{~mm}$, maximum pain).

- The ROM of active knee flexion was measured using a goniometer. ${ }^{18}$ Goniometry assessment procedures are one of the most common procedures in the daily clinical practice. Furthermore, the use of the universal goniometer has shown to have a good inter and intra observer reliabitliy. ${ }^{19}$ For the measurements, the patients were placed in supine lying with the legs outstretched. Subsequently, the patient was asked to perform knee flexion until the appearance of pain in the anterior aspect of the knee, which reminded the person of their pain. Special care was taken to avoid the patient reaching a ROM of flexion until the maximum pain possible, to avoid triggering a knee aggravation which could affect subsequent measurements. An examiner with over 5 years' experience in goniometry performed three measurements, selecting the mean of these as the final value.

All the variables were measured before, immediately after, and 24 hours after the intervention.

\section{Procedure}

All subjects received a single intervention of US-guided PNM on the femoral nerve. Patients were placed in supine lying with the anterior aspect of the hip uncovered. The intervention consisted of the application of a biphasic square wave electric current, with a frequency of $10 \mathrm{~Hz}$, a phase duration of $250 \mu \mathrm{s}$, and a maximum tolerable intensity to cause an exacerbated muscle contraction for 1.5 minutes in total, according to the protocol by Valera-Garrido and MinayaMuñoz. ${ }^{12} \mathrm{~A}$ health-certified device (Physio Invasiva, PRIM, Móstoles, Madrid, Spain) was used. The femoral nerve was located at the cross-section of the groin crease, using an ultrasound device with a high-frequency 12L linear probe (transverse section; Logiq e, GE Healthcare, Chicago, IL, US). Subsequently, a stainless steel acupuncture needle $(0.30 \mathrm{~mm}$ x $30 \mathrm{~mm}$; Physio Invasiva, PRIM) was inserted into the short axis, with a $80^{\circ}$ tilt from the skin surface, until the perineurium of the femoral nerve (-Fig. 1) Before inserting the needle, the patient's skin was cleaned with a 70\% isopropyl alcohol based solution. A physical therapist with 6 years of experience in invasive physical therapy administrated the intervention.

All patients were asked to report any adverse event experienced during the research.

\section{Statistical Analysis}

For the statistical analysis, the Statistical Package for the Social Sciences (SPSS, IBM Corp., Armonk, NY, US) software, version 21.0 for MAC OS was used. The normality of the sample distribution was evaluated using the Shapiro-Wilk test. For the descriptive analysis, the results were expressed as means and standard deviations. Likewise, for the comparison between the different measurement moments (M1: preintervention; M2: postintervention; and M3: 24 hours 


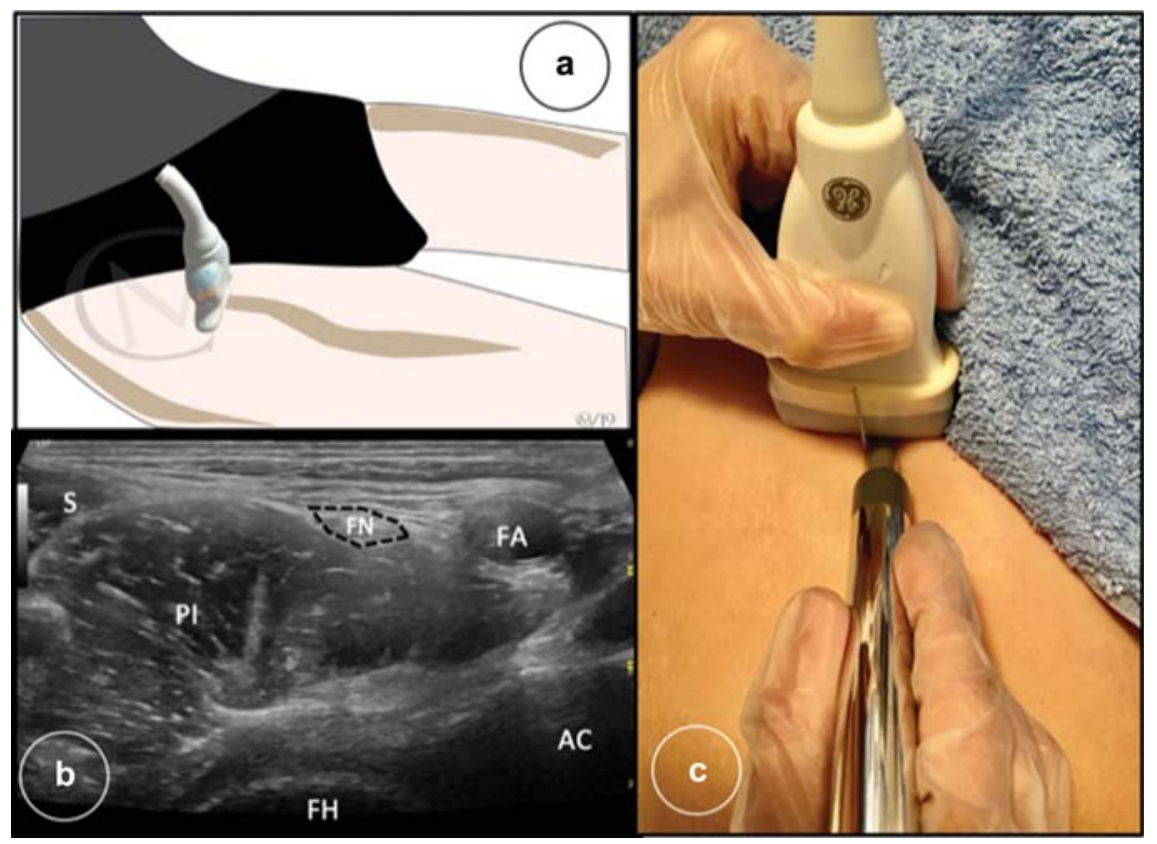

Fig. 1 US-guided percutaneous neuromodulation. (a) Placement of the probe for sonographic visualization of the femoral nerve; (b) ultrasound image of the intervention; (c) intervention on the femoral nerve. Abbreviations: AC, acetabulum; FA, femoral artery; FH, femoral head; FN, femoral nerve; PI, psoas-iliacus; S, sartorius muscle.

after the intervention), the Student's $t$-test was used for related samples, considering a significance level of $p<0.05$.

The effect size (ES; $90 \%$ confidence interval [ $90 \% \mathrm{CI}]$ ) was calculated for the selected variables. ${ }^{20}$ The threshold values to calculate the magnitude of the ES were $<0.20,0.20-0.59$, $0.60-1.2$ and $>1.2$ for trivial, small, moderate and large, respectively. The qualitative changes of greater to lesser magnitude were quantitatively evaluated as follows: $<1 \%$, almost certainly 'no'; $1-5 \%$, very unlikely; $5-25 \%$, unlikely; 25-75\%, possible; 75-95\%, likely; 95-99\%, very likely; > 99\%, almost certain. ${ }^{21}$ A substantial effect was considered $>75 \% .{ }^{21}$

Finally, the inter-examiner reliability was calculated via the interclass correlation coefficient (ICC), the standard error of measurement (SEM) and the minimal detectable change (MDC) at 95\% for the knee flexion ROM.

\section{Results}

- Table 1 displays the sociodemographic variables of the sample, including age ( $36 \pm 5.76$ years), weight $(65.37 \pm 8.48$ $\mathrm{Kg})$, height $(164 \pm 0.05 \mathrm{~cm})$, BMI $(23.96 \pm 1.69)$, duration of symptoms ( $5.81 \pm 0.53$ months). We recruited six women and two men, all of whom had pain in the right knee.

-Table 2 displays the values of the flexion ROM and the level of pain at the beginning of the study, after the intervention, and 24 hours after the same. Compared with the baseline measurements, differences were observed in the level of pain immediately after the intervention (likely; ES: $-1.30 \pm 1.14$ ) and after 24 hours (almost certain; ES: $-1.76 \pm 0.80)$. However, the percentage of change was greater at 24 hours $(-71.4 \%)$ than immediately after the intervention (14.3\%). Regarding the knee flexion ROM, a significant difference was only observed 24 hours after the intervention
Table 1 Sociodemographic data of the sample

\begin{tabular}{|l|l|}
\hline Variables & $\begin{array}{l}\text { Mean } \pm \text { standard } \\
\text { deviation }\end{array}$ \\
\hline Age (years) & $36 \pm 5.76$ \\
\hline Weight $(\mathrm{kg})$ & $65.37 \pm 8.48$ \\
\hline Height $(\mathrm{cm})$ & $164 \pm 0.05$ \\
\hline Body mass index $\left(\mathrm{kg} / \mathrm{m}^{2}\right)$ & $23.96 \pm 1.69$ \\
\hline Duration of symptoms (months) & $5.81 \pm 0.53$ \\
\hline Gender (male/female) & $6 / 2$ \\
\hline Dominant side (right/left) & $8 / 0$ \\
\hline Affected side (right/left) & $8 / 0$ \\
\hline
\end{tabular}

(almost certain; ES: $1.73 \pm 0.44)$. However, the percentage of change was greater at 24 hours (15.2\%) (- Table 2, - Fig 2).

Finally, the measurement of the knee flexion ROM showed a high inter-examiner reliability $(\mathrm{ICC}=0.98$ ). No adverse effects were registered, neither during the intervention nor during the follow-up period.

\section{Discussion}

The main finding of this study was that an improvement was obtained for both variables under study (ROM and pain), which was greater at 24 hours after the stimulation. Concretely, a decrease in pain and increased ROM of knee flexion was observed, therefore knee mobility improved (-Table $\mathbf{2}$ ). These results confirmprevious studies ${ }^{22,23}$ which focused on therapeutic benefits after the application of US-guided PNM.

Neuromodulation consists of the application of an electric current to provide pain relief. Although the physiological 
Table 2 Level of pain and knee flexion range of motion before (M1), immediately after (M2) and 24 hours (M3) after the intervention

\begin{tabular}{|c|c|c|c|c|c|c|c|}
\hline & \multicolumn{3}{|l|}{ Measurements } & \multirow{2}{*}{$\begin{array}{l}\text { Standardized } \\
\text { differences and } \\
\text { QA }(90 \% \mathrm{Cl}) \\
\text { (M1 versus M2) }\end{array}$} & \multirow{2}{*}{$\begin{array}{l}\text { Mean } \\
\text { change (\%) } \\
\text { (M1 versus } \\
\text { M2) }\end{array}$} & \multirow{2}{*}{$\begin{array}{l}\text { Standardized } \\
\text { differences } \\
\text { and QA } \\
\text { (90\%Cl) } \\
\text { (M1 versus } \\
\text { M3) }\end{array}$} & \multirow{2}{*}{$\begin{array}{l}\text { Mean change (\%) } \\
\text { (M1 versus M3) }\end{array}$} \\
\hline & $\begin{array}{l}\text { Pre-intervention } \\
\text { (M1) }\end{array}$ & $\begin{array}{l}\text { Post-intervention } \\
\text { (M2) }\end{array}$ & $\begin{array}{l}\text { After } \\
24 \text { hours } \\
\text { (M3) }\end{array}$ & & & & \\
\hline $\begin{array}{l}\text { Range of } \\
\text { motion }\left({ }^{\circ}\right)\end{array}$ & $\begin{array}{l}135.5 \pm 5.97 \\
(131.4 ; 139.6)\end{array}$ & $\begin{array}{l}139.7 \pm 5.11^{\mathrm{a}} \\
(136.2 ; 143.2)\end{array}$ & $\begin{array}{l}147.1 \pm 4.45^{\mathrm{b}} \\
(144.0 ; 150.2)\end{array}$ & $\begin{array}{l}0.63 \pm 0.43 \\
(95 / 4 / 0) ; \\
\text { very likely }\end{array}$ & 10.4 & $\begin{array}{l}1.73 \pm 0.44^{* *} \\
(100 / 0 / 0) \\
\text { almost certain }\end{array}$ & 15.2 \\
\hline $\begin{array}{l}\text { Level of } \\
\text { pain }(\mathrm{mm})\end{array}$ & $\begin{array}{l}64.3 \pm 14.9 \\
(54.0 ; 74.6)\end{array}$ & $\begin{array}{l}42.5 \pm 28.1^{a} \\
(23.0 ; 62.0)\end{array}$ & $\begin{array}{l}34.7 \pm 20.8^{b} \\
(20.3 ; 49.1)\end{array}$ & $\begin{array}{l}-1.30 \pm 1.14^{*} \\
(2 / 3 / 94) ; \\
\text { probable }\end{array}$ & -14.3 & $\begin{array}{l}-1.76 \pm 0.80^{* *} \\
(0 / 0 / 100) ; \\
\text { almost certain }\end{array}$ & -71.4 \\
\hline
\end{tabular}

Abbreviations: $\mathrm{Cl}$, confidence interval; $\mathrm{QA}$, qualitative assessment.

Notes: The data are expressed as mean \pm standard deviations, with $90 \%$ confidence intervals.

${ }^{a}$ Substantial difference between the preintervention and postintervention values.

bubstantial difference between preintervention values and the values after 24 hours.

${ }^{*} p<0.05$, statistically significant difference between the preintervention and postintervention values.

${ }^{* *} p<0.001$, statistically significant difference between the preintervention values and the values after 24 hours.

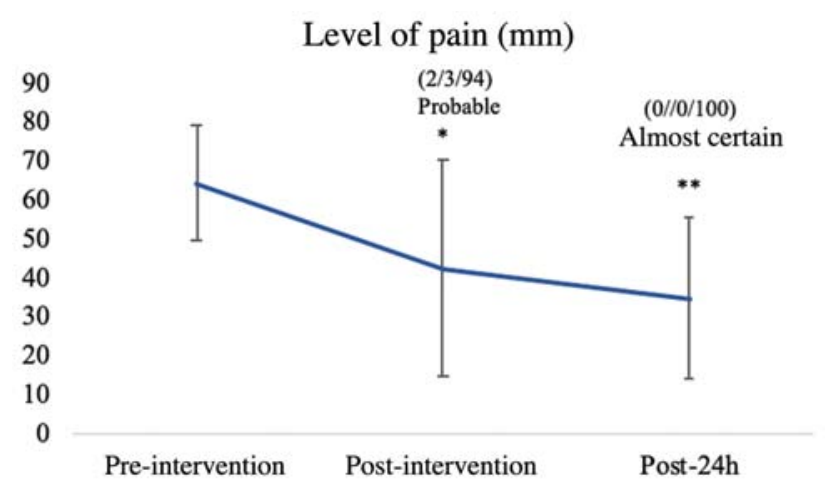

Flexion ROM ( $\left.{ }^{\circ}\right)$

160

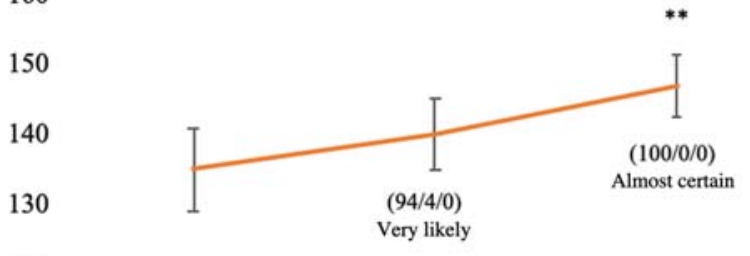

120

110

Pre-intervention Post-intervention Post-24h

Fig. 2 Pain scores and knee flexion ROM for each of the measurements. ${ }^{*} p<0.05$, statistically significant difference between the preintervention and postintervention values. ${ }^{* *} p<0.001$, statistically significant difference between the preintervention values and the values after 24 hours.

mechanism is not well known, it is suggested that this is related to the gate-control theory proposed by Melzack and Wall. ${ }^{24}$ This theory states that any intervention that activates the large-diameter mechanosensitive afferents has the potential to modulate the transmission of pain in the spinal cord. ${ }^{24}$ Currently, the use of percutaneous neuromodulation has been rapidly increasing, while undergoing clinical research to determine the most appropriate methodology. Indeed, De-la-
Cruz-Torres et $\mathrm{al}^{25}$ demonstrated that percutaneous applications produce a greater qualitative improvement of performance compared to transcutaneous applications after the stimulation of the flexor halluxis longus muscle in ballerinas.

The application of US-guided PNM has demonstrated to be an effective procedure, which is both easy to perform and safe. Positive effects have been obtained upon completion of treatment for the reduction of pain ${ }^{23,26}$ and re-establishment of strengh. ${ }^{27}$ Ilfeld et al. ${ }^{26}$ obtained a decrease of pain and an increase of passive and active ROM of knee flexion during the application of the current in patients with post-surgical pain. Also, Ilfeld et al. ${ }^{23}$ found a decrease of pain after a treatment of US-guided PNM on the femoral nerve in patients with reconstruction of the anterior cruciate ligament. Besides, ÁlvarezPrats et al. ${ }^{27}$ demonstrated an immediate increase in isometric quadriceps strength after the stimulation of the femoral nerve in patients with knee pain. The findings of this study suggest that, the percentage of change is greater at 24 hours after a single intervention. According to the authors, no previous study has analyzed the short-term effect of a single intervention using US-guided PNM, beyond the immediate effects or post-treatment effects. Therefore, the relevance of these preliminary data for clinical practice is that this study adds information regarding the duration of the effect of US-guided PNM treatment, in order to determine the correct application of the same with greater precision. Due to the clinical benefits of this technique, further studies are required to examine the methodological principles of this technique and discover its possible indications.

As in previous studies, ${ }^{19,28}$ a high inter-evaluator reliability for the knee flexion ROM was observed. Besides, the SEM and the $\mathrm{MDC}(90 \% \mathrm{CI})$ had values of 1.81 and 1.77 respectively, immediately after, and values of 1.57 and 1.54 respectively, 24 hours after the stimulation.

This study presents limitations. In the first place, the reduced size of the sample and the absence of a control group. Secondly, the lack of follow up in the mid and long term. Finally, this study did not consider psychosocial aspects such as fear of 
movement or pain catastrophizing, as this study measured the effects of the intervention in the short term (two days) and therefore it was not foreseeable for psychosocial aspects to change during the study.

\section{Conclusion}

A single intervention of US-guided PNM on the femoral nerve produces a decrease of the AKP and an increased range of flexion, greater at 24 hours after stimulation.

\section{Conflict of Interests}

The authors have no conflict of interests to declare.

\section{References}

1 Aglietti P, Buzzi R, Pisaneschi A. Patella pain. J Sports Trauma Rel Res. 1990;12:131-150

2 Goldberg B. Chronic anterior knee pain in the adolescent. Pediatr Ann 1991;20(04):186-187, 190-193

3 Sanchis-Alfonso V, Dye SF. How to deal with anterior knee pain in the active young patient. Sports Health 2017;9(04):346-351

4 Sanchis-Alfonso V. Holistic approach to understanding anterior knee pain. Clinical implications. Knee Surg Sports Traumatol Arthrosc 2014;22(10):2275-2285

5 Günther KP, Thielemann F, Bottesi M. [Anterior knee pain in children and adolescents. Diagnosis and conservative treatment]. Orthopade 2003;32(02):110-118

6 Fairbank JC, Pynsent PB, van Poortvliet JA, Phillips H. Mechanical factors in the incidence of knee pain in adolescents and young adults. J Bone Joint Surg Br 1984;66(05):685-693

7 Lin PL, Shih YF, Chen WY, Ma HL. Neurodynamic responses to the femoral slump test in patients with anterior knee pain syndrome. J Orthop Sports Phys Ther 2014;44(05):350-357

8 de la Cruz Torres B. Strength-duration curve of radial nerve in patients with lateral elbow pain. J Sport Rehabil 2019;18:1-6

9 Rodríguez-Fernández ÁL, Rebollo-Roldán J, Jiménez-Rejano JJ, Güeita-Rodríguez J. Strength-Duration curves of the common fibular nerve show hypoexcitability in people with functional ankle instability. PM R 2016;8(06):536-544

10 White JC, Sweet WH. Pain and the neurosurgeon: a forty-year experience. Springfield: Thomas; 1969:894-899

11 Stuart RM, Winfree CJ. Neurostimulation techniques for painful peripheral nerve disorders. Neurosurg Clin N Am 2009;20(01): 111-120, vii-viii

12 Valera-Garrido F, Minaya-Muñoz F. Fisioterapia Invasiva. Barcelona: Elsevier España SL; 2016

13 De-la-Cruz-Torres B. Ultrasound-guided Percutaneous neuromodulation in patients with unilateral refractory lateral epicondylitis. J Manipulative Physiol Ther 2019; In press

14 Rossi M, DeCarolis G, Liberatoscioli G, Iemma D, Nosella P, Nardi LF. A novel mini-invasive approach to the treatment of neuropathic pain: the PENS study. Pain Physician 2016;19(01): E121-E128
15 de la Cruz-Torres B, Barrera-García-Martín I, Albornoz-Cabello M. Immediate effects of ultrasound-guided percutaneous neuromodulation versus physical exercise on performance of the flexor hallucis longus muscle in professional dancers: a randomised clinical trial. Acupunct Med 2019;37(02):91-97

16 Recoules-Arché D, Gayet A. [L4 crural neuralgia and disc hernia of the L4-L5 intervertebral foramen]. Rev Rhum Mal Osteoartic 1986;53(03):161-165

17 Hjermstad MJ, Fayers PM, Haugen DF, et al; European Palliative Care Research Collaborative (EPCRC). Studies comparing Numerical Rating Scales, Verbal Rating Scales, and Visual Analogue Scales for assessment of pain intensity in adults: a systematic literature review. J Pain Symptom Manage 2011;41(06):1073-1093

18 Norkin C, White DJ. Goniometría, evaluación de la movilidad articular. Marban; 2007

19 Brosseau L, Tousignant M, Budd J, et al. Intratester and intertester reliability and criterion validity of the parallelogram and universal goniometers for active knee flexion in healthy subjects. Physiother Res Int 1997;2(03):150-166

20 Hopkins WG, Marshall SW, Batterham AM, Hanin J. Progressive statistics for studies in sports medicine and exercise science. Med Sci Sports Exerc 2009;41(01):3-13

21 Suarez-Arrones L, Arenas C, López G, Requena B, Terrill O, Mendez-Villanueva A. Positional differences in match running performance and physical collisions in men rugby sevens. Int J Sports Physiol Perform 2014;9(02):316-323

22 Ilfeld BM, Gilmore CA, Grant SA, et al. Ultrasound-guided percutaneous peripheral nerve stimulation for analgesia following total knee arthroplasty: a prospective feasibility study. J Orthop Surg Res 2017;12(01):4

23 Ilfeld BM, Said ET, Finneran JJ IV, et al. Ultrasound-Guided Percutaneous Peripheral Nerve Stimulation: Neuromodulation of the Femoral Nerve for Postoperative Analgesia Following Ambulatory Anterior Cruciate Ligament Reconstruction: A Proof of Concept Study. Neuromodulation 2019;22(05):621-629

24 Melzack R, Wall PD. Pain mechanisms: a new theory. Science 1965;150(3699):971-979

25 de-la-Cruz-Torres B, Barrera-García-Martín I, Romero-Morales C. Comparative Effects of One-Shot Electrical Stimulation on Performance of the Flexor Hallucis Longus Muscle in Professional Dancers: Percutaneous Versus Transcutaneous? Neuromodulation 2019; In press

26 Ilfeld BM, Grant SA, Gilmore CA, et al. Neurostimulation for Postsurgical Analgesia: A Novel System Enabling UltrasoundGuided Percutaneous Peripheral Nerve Stimulation. Pain Pract 2017;17(07):892-901

27 Álvarez-Prats D, Carvajal-Fernández O, Pérez-Mellada N, MinayaMuñoz F. Changes in Maximal Isometric Quadriceps Strength after the Application of Ultrasound-Guided Percutaneous Neuromodulation of the Femoral Nerve: A Case Series. Rev Fisioter Invasiva 2019;2:39-45

28 Brosseau L, Balmer S, Tousignant M, et al. Intra- and intertester reliability and criterion validity of the parallelogram and universal goniometers for measuring maximum active knee flexion and extension of patients with knee restrictions. Arch Phys Med Rehabil 2001;82(03):396-402 